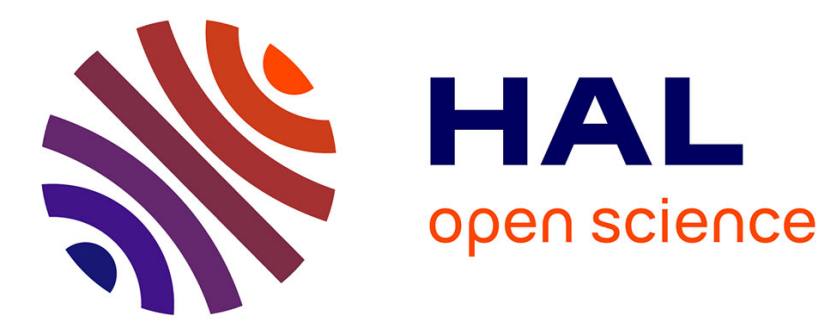

\title{
L'évolution du statut de la connaissance dans le traité du serf-arbitre de Luther 1
}

Charles T. Wolfe, Fabrice Stroun

\section{To cite this version:}

Charles T. Wolfe, Fabrice Stroun. L'évolution du statut de la connaissance dans le traité du serf-arbitre de Luther 1. Archives de Philosophie, 2003, 66 (2), pp.279-302. hal-01238106

\section{HAL Id: hal-01238106 \\ https://hal.science/hal-01238106}

Submitted on 20 Feb 2019

HAL is a multi-disciplinary open access archive for the deposit and dissemination of scientific research documents, whether they are published or not. The documents may come from teaching and research institutions in France or abroad, or from public or private research centers.
L'archive ouverte pluridisciplinaire HAL, est destinée au dépôt et à la diffusion de documents scientifiques de niveau recherche, publiés ou non, émanant des établissements d'enseignement et de recherche français ou étrangers, des laboratoires publics ou privés. 


\title{
L'évolution du statut de la connaissance dans le traité du serf-arbitre de Luther ${ }^{1}$
}

\author{
CHARLES T. WOLFE \& FABRICE STROUN \\ Université de Toulouse, JRP Editions - Genève
}

A la mémoire de Reiner Schürmann

L'essai qui suit se serait présenté sous des airs encore plus pesants, s’il s'était déclaré comme une "approche gnoséologique»du Traité du serfarbitre. C'est bien au thème de la connaissance, pourtant, que nous nous consacrerons. Mais ainsi que l'indique notre titre, nous mettrons plutôt en valeur l'aspect des modalités, des types de connaissance, ou de sa progression. En d'autres termes, c'est une tentative d'analyser la manière dont la connaissance apparaît dans l'œuvre de Luther, et comment il en résoud le "problème ». Les modalités, les types, la progression : trois expressions qui désignent, chacune à sa façon, le «destin » de la connaissance. Est-elle un tout qui peut être décomposé en diverses modalités? Ou, de manière plus statique, une série de types qui pourront être décrits au moyen d'une typologie? Enfin, serait-elle un genre de chemin qui comprendrait des étapes dans une évolution progressive - en d'autres termes, un multiple plutôt que l'unité d'un tout? On notera que l'idée d'un chemin, d'une « voie» ou d'un "parcours" ne sous-entend pas forcément une idée de développement, pas plus qu'elle ne se définit comme une progression logique ou simplement chronologique. Pour finir, au risque de sembler vouloir dissoudre l'existence indépendante de ces catégories avant même qu'elles ne soient venues à être, il faut mentionner la probabilité que, dans un vocabu-

1. Deux traductions du Traité du serf-arbitre [1525] seront utilisées : l'édition des Euvres, trad. Jean Carrère, Genève, Labor et Fides, 1957..., tome V, et la nouvelle traduction par Georges Lagarrigue, Gallimard, 2000, citée G, avec entre parenthèses les références au tome XVIII de l'édition de Weimar des œuvres de Luther (Martin Luthers Werke. Kritische Gesamtausgabe, Weimar, 1883...). - Nous remercions Felix Ensslin, qui a beaucoup fait pour ce travail. 
laire contemporain, ces catégories soient "floues » ou "poreuses », ou, en termes plus nets, qu'elles soient corrélatives : elles doivent toutes être invoquées ensemble ou interpellées les unes avec les autres afin de décrire les apparences de la connaissance dans ses entrelacements et distanciations avec la foi.

\section{LA GONNAISSANGE DE L'ÉGRITURE}

\section{La clarté du ministère de la Parole}

Contre Érasme, lequel affirme trouver «peu de satisfaction » dans «les affirmations » de Luther, et ajoute qu'il suivrait "volontiers l'opinion des sceptiques, partout où cela est admis par l'autorité inviolable de l'Écriture sainte et les décrets de l'Église » (Traité du serf-arbitre, p. 23 [603]) ${ }^{2}$, Luther établit la nécessité d'une théologie doctrinale, pour les «choses que nous devons affirmer, aux choses que Dieu nous a transmises dans l'Écriture sainte » (ibid.). L'Écriture est ainsi posée dès le départ comme étant absolument claire : «,...] il ne reste rien d'obscur ni d'ambigu, mais tout ce qui se trouve dans l'Écriture a été mis en pleine lumière par la Parole et annoncé publiquement au monde entier» (p. 29 [609]). Pour Luther, l'Écriture contient l'affirmation directe ou « simple confession » (p. 81G [608]) de faits entièrement intelligibles - le "contenu » de l'Écriture. Les affirmations directes de ces faits constituent le ministère de la Parole : la Parole révélée de Dieu telle que rapportée dans l'Évangile prêché au monde entier. La théologie doctrinale est l'affirmation du ministère de la Parole, la formulation et l'organisation systématique du contenu de l'Écriture.

Les doctrines chrétiennes sont alors comprises comme n'étant rien de plus que l'énonciation pure et simple des faits qui constituent le contenu de l'Écriture, lesquels sont sans ambiguïté, per definitionem. Ainsi, non seulement l'Écriture possède-t-elle une autorité unique et absolue sur toutes les questions que l'on pourrait se poser sur la vérité et la fausseté de tout point de doctrine, mais aussi, elle comprend et exige simultanément l'affirmation d'une réponse unique. C'est pourquoi Luther considère comme un blasphème l'énoncé d'Érasme selon lequel certaines doctrines seraient « mystérieuses » (p. 28 [607]). Une fois la clarté de l'Écriture admise, se poser contre les affirmations, comme le fait Érasme, qui se range du côté des sceptiques, c'est donc se poser contre la chrétienté toute entière.

2. Dorénavant les pages du Traité du serf-arbitre seront données directement dans le texte sans autre mention. 


\section{La connaissance du cœur}

«La clarté de l'Écriture est double, tout comme est double son obscurité. L'une est extérieure et réside dans le ministère de la Parole ; l'autre est intérieure et se situe dans la connaissance qui est celle du cœur » (p. 82G [609]).

La clarté de la Parole ne suffit pas à percevoir sa "très claire vérité " (p. 80G [607]). Ce qui est exigé est une «connaissance du cœur », puisque « aucun homme ne peut percevoir un iota dans l'Écriture, s'il n'a pas l'Esprit de Dieu » (p. 29 [609]). Tout comme le ministère de la Parole, la perception interne de sa clarté absolue est un don de Dieu. Comme nous le verrons plus loin, cette perception ou reconnaissance interne se situe à l'intérieur de la foi, laquelle, pour Luther, est un don de Dieu, une grâce divine : elle est le sceau du ministère de la Parole apposé par la lumière salvatrice du SaintEsprit sur le cœur du croyant. "En effet, l'Esprit est nécessaire pour comprendre toute l'Écriture, ou n'importe quelle partie de l'Écriture» (ibid.).

Ce qui importe ici est que la clarté de l'Écriture est à deux niveaux : d'une part, le niveau externe, la représentation extérieure de la clarté, grâce à laquelle nous obtenons une clarté absolue de la Parole, et d'autre part, le niveau interne, la représentation intérieure de la clarté, par laquelle nous reconnaissons cette clarté absolue. Dès lors, comment une chose peut-elle être absolument claire et réclamer malgré tout une condition spéciale donnée par Dieu - à celui qui la perçoit (ou qui l' " entend », puisqu'il s'agit de la Parole), afin d'être appréhendée ? En d'autres termes, comment ces deux niveaux de la clarté de la Parole, interne et externe, interagissent-ils, comment communiquent-ils?

Afin de répondre à ces questions, il faut revenir sur l'argument premier de Luther contre Érasme, au sujet des " affirmations théologiques ». L'attaque de Luther oscille sans cesse entre deux thèses. La première concerne la différenciation nécessaire entre la connaissance de la volonté divine et la connaissance de sa Parole ; la seconde concerne la différenciation nécessaire entre connaissance et compréhension rationnelle. L'existence de la clarté de l'Écriture sur deux niveaux (interne et externe) permet un balancement constant entre la question : "que savons-nous?", à laquelle la réponse est «La Parole de Dieu telle qu'elle nous est révélée dans l'Évangile à travers Jésus-Christ ", et la question : "comment le savons-nous?", à laquelle la réponse est : "par la lumière du Saint-Esprit ». Un balancement entre un objet extérieur - la clarté du ministère de la Parole - et l'événement permettant de connaître ce même objet de l'intérieur. Ces deux questions coexistent dans un état d'interdépendance et de réciprocité. Certes, une partie de la difficulté provient du fait que le premier terme de ce rapport 
produit les conditions de possibilité pour l'émergence du second, alors que le second est essentiellement différent du premier. De plus, le mouvement de va-et-vient de l'argumentation de Luther contre Érasme, entre (a) la différenciation entre la connaissance de la volonté divine et sa Parole et (b) la différenciation entre la connaissance et la compréhension rationnelle, suit le balancement des deux questions mentionnées plus haut.

\section{LA GONNAISSANGE NATURELLE}

\section{Dieu est prêché et n'est pas prêché}

Luther accuse Érasme de confondre la connaissance de la volonté infinie de Dieu et la connaissance de l'Écriture. "Il y a deux choses : Dieu et l'Écriture ; de même qu'il y a aussi deux choses : le Créateur et la créature " (p. 27 [606]). D'une part il y a la clarté complète de l'Écriture ; de l'autre il y a la volonté qui a nécessité ce contenu en tant que tel. Tout ce qui est donné à connaître à l'homme, c'est le ministère de la Parole, c'est-à-dire, ce que Dieu a permis à l'homme de connaître de lui-même et de sa volonté, à travers le Christ et l'Évangile : «L'Écriture affirme simplement et clairement la Trinité de Dieu, l'incarnation du Christ et le péché irrémissible. Il n'y a là ni obscurité ni ambiguïté. Mais l'Écriture - contrairement à ce que tu imagines - ne dit pas comment ces choses se passent, et il ne nous est pas nécessaire de le savoir » (p. 29 [608]).

Comme les œuvres de la volonté divine ne sont pas révélées par les Écritures, il n'est pas légitime pour l'homme de tenter de les comprendre de pénétrer les «secrets de sa majesté »(p. 279G [706]), " en un endroit où "ses jugements sont incompréhensibles" (Romains 11:33)" (p. 292G [712]) . Une telle enquête équivaudrait à chercher à connaître Dieu indépendamment de sa volonté et de ses dons (l’Écriture et la grâce). Comme nous le verrons plus loin, cette affirmation de l'indépendance de l'homme dans le processus de la connaissance de Dieu est l'un des plus grands blasphèmes pour Luther.

Derrière le caractère purement théologique de ce débat, et de la condamnation d'Érasme par Luther, il importe de noter que, dès le début du Traité, Luther affirme que la Parole révélée de Dieu est la condition nécessaire qui permet toute connaissance de Lui et de tout ce qui est lié au salut; et ce "permet" a le sens à la fois de "poser » et d' "engendrer». De plus, la différentiation luthérienne entre Dieu prêché (le contenu de l'Écriture) et non prêché (sa volonté) établit une différenciation entre la connaissance et la compréhension rationnelle. 


\section{La connaissance n'est pas la compréhension rationnelle}

Bien que l'homme puisse connaître le contenu clair de l'Écriture, il ne peut rationnellement comprendre la volonté qui réclame un tel contenu. Soyons clairs : la raison pour laquelle l'homme ne peut rationnellement comprendre la volonté divine n'est pas que l'entendement rationnel soit insuffisamment "puissant " pour traverser les œuvres de la volonté divine, ou que l'activité finie de la raison ne puisse tout simplement pas saisir la toute-puissance de la volonté divine. Luther comprend la raison comme une faculté essentiellement humaine qui, en tant que telle, n'est pas seulement limitée par définition, mais est aussi réfractaire à la volonté divine ${ }^{3}$. De sorte que l'usage de cette faculté "hors" des dons de Dieu peut seulement éloigner l'homme de Dieu, de la vraie connaissance de la Parole et de la volonté divine, vers un "rêve " impossible et blasphématoire. D'ailleurs, comme nous le verrons plus loin, la "raison » pratiquée à l'intérieur des dons de Dieu ne peut plus être considérée comme la "Raison ».

Ainsi, quand Érasme affirme que certaines doctrines chrétiennes présentent des problèmes qui n'ont jamais été résolus (la distinction des Personnes, « l'union étroite de la nature divine et de la nature humaine, du péché irrémissible », p. 81G [608]), il est renvoyé aux « rêves des sophistes » (p. 29 / $82 \mathrm{G}[609])$. Les sophistes rêvent de comprendre les secrets de la volonté divine plutôt que le contenu même de l'Écriture. Pour Luther, les « sophistes », ce sont les théologiens qui maintiennent un idéal d'autonomie rationnelle et d'autosuffisance de la raison. On l'a vu, la thèse affirmant que la connaissance de Dieu peut être atteinte par le recours à la seule raison, c'est-à-dire sans que la raison soit secourue par Dieu, est à la fois blasphématoire en principe - car ceux qui affirment cette idée cherchent à connaître Dieu indépendamment de sa volonté et de ses dons (l'Écriture et la grâce) et une défaite en pratique, puisque cela donne lieu à des spéculations sans fin (lesquelles sont elles-mêmes propres à l'intellect humain, qui est curvatus in se) qui éloignent du ministère de la Parole : « la raison humaine [...] interprète d'après ses propres déductions et syllogismes les Écritures de Dieu, et les tire dans la direction qu'elle veut » (p. 203G [673]), c'est-à-dire loin de la doctrine.

On ne peut pas connaître la volonté de Dieu par la compréhension rationnelle, car un objet de la raison est un objet pour la raison, fabriqué par la raison. La compréhension rationnelle est indépendante à la fois (i) de la volonté de Dieu, dont elle tente une appréhension rationnelle qui transfor-

3. Nous reviendrons à cette notion d'une prédisposition naturellement pécheresse associée aux facultés humaines, en d'autres termes, à l'asservissement de la volonté humaine à la volonté de Satan. 
merait la volonté de Dieu prêché et l'Écriture en objets rationnels, et (ii) de la manière dont elle appréhende l'objet, autrement dit, des facultés ou capacités cognitives de celui qui appréhende (tout ce qui compte, c'est : la personne a-t-elle la foi ou non ?). Il y a précisément trop d'activité dans la raison, dira Luther : trop d'activité chez le sujet rationnel. L'illusion qu'a l'homme de posséder le libre arbitre naît de cette activité « aveuglante ».

La raison fabrique son objet, nécessite son objet, par un acte de volonté : il y a une volonté de raisonner, une volonté de la raison, qui remplace la volonté divine. Puisque son activité aveuglante et indépendante produit continuellement sa nécessité illusoire, il est impossible pour la raison naturelle de reconnaître rationnellement que Dieu nécessite toutes choses ; il lui est impossible de connaître rationnellement sa Volonté.

La raison naturelle est ainsi naturellement réfractaire à l'affirmation suivant laquelle Dieu nécessite toutes choses. Le sujet rationnel, dans son activité, ne peut cesser de produire l'illusion de son propre libre-arbitre, résistant ainsi à l'affirmation que la volonté divine nécessite toutes choses. $\mathrm{Ou}$, comme l'activité de la raison naturelle obscurcit sa connaissance de la volonté infinie de Dieu, le sujet rationnel est laissé sans aucune autre option que celle d'affirmer qu'il (le sujet) nécessite certaines choses, i.e., le sujet rationnel affirme qu'il est, jusqu'à un certain point, son propre principe. Ce «certain point "s'élève nécessairement jusqu'à la prétention que l'homme est ou possède en lui-même son propre principe, sans qualification. Cela ne va pas seulement à l'encontre de la doctrine de la chute, mais obscurcit l'étendue totale, à la fois de la servitude de l'homme en tout ce qui touche au salut (sotériologie), et de la foi et de la grâce.

Cette revendication d'indépendance, d'autonomie, atteste de l'extériorité absolue de la connaissance rationnelle et du sujet rationnel à l'objet prétendument « connu », à savoir, la volonté divine.

L'objet fabriqué par la raison naturelle, c'est-à-dire le libre-arbitre de l'homme, est une illusion. En tout ce qui concerne la connaissance de Dieu et le salut, l'homme ne nécessite rien, ne possède aucun libre-arbitre : « s'agissant de Dieu ou des choses qui concernent le salut ou la damnation, l'homme n'a pas le libre-arbitre, mais il est captif, sujet et esclave soit de la volonté de Dieu, soit de la volonté de Satan » (p. 131G [638]). L'homme - la volonté humaine - est comme une bête de somme placée entre deux cavaliers : "Si c'est Dieu qui la monte, elle veut aller et elle va là où Dieu veut [...]. Si Satan la monte, elle veut aller et elle va là où Satan veut » (p. 127G [635]).

L'homme est naturellement déchu, sous la domination de la volonté de Satan; c'est sa caractéristique "charnelle». (On remarquera que cette notion de "charnellité » dépasse de loin toute doctrine traditionnelle de la concupiscentia, et signifie plutôt l'autonomie absolue de l'individu, en 
dehors de la grâce, totalement extérieure à la volonté divine. Son activité est toujours et nécessairement dirigée contre Dieu, qu'il soit «prêché » ou non.) Puisque la volonté de Satan est évidemment nécessitée par Dieu, la volonté humaine est ou bien naturellement condamnée par la volonté divine ou bien exceptionnellement sauvée par sa grâce. Mais l'homme ne peut rien affirmer sur cette question via la raison, car cette structure appartient à la volonté divine (Dieu non prêché). Nous connaissons le fait de la chute via l'Évangile, mais sa structure et sa dynamique ne nous sont pas accessibles. L'activité de la raison naturelle est nécessitée naturellement par le cœur naturel et charnel de l'homme.

Qu'est-ce que cela signifie, sinon que le libre-arbitre ou le «cœur de l'homme » est tellement lié au pouvoir de Satan, opprimé et ainsi délimité par lui, qu'à moins d'être miraculeusement " accru ", " animé » ou "éveillé " par l'Esprit de Dieu, il ne peut pas par lui-même « voir ni entendre les choses qui frappent manifestement les yeux et les oreilles, si bien qu'on pourrait les toucher avec la main » (p. 171G [658])?

Sans intervention divine, donc à moins que son cœur ne soit «animé » par le Saint-Esprit, l'homme emploie naturellement et nécessairement l'instrument de la raison. Le cours de la raison naturelle se détourne de la véritable connaissance de la volonté divine, et fabrique la confusion et l'aveuglement. Le mystère de la volonté divine requiert alors de l'homme, non pas une activité d'enquête rationnelle, mais son adoration, c'est-à-dire un comportement passif plutôt qu'un comportement actif : "Il ne nous appartient pas de chercher à connaître cela [sc. la volonté divine, les « secrets de la majesté »], mais d'adorer ces mystères » (p. 292G [712]).

Cependant, l'attaque de Luther contre la raison naturelle n'est ni un appel à la démence ni un appel à l'idiotie (ce sont les sophistes qui sont traités de "déments» ou d' «idiots»). Encore moins cette attaque est-elle mystique : l' «adoration » exigée ne détruit pas la rationalité, puisque la théologie doctrinale, formulation et organisation systématique du contenu de l'Écriture, est une activité rationnelle.

Deux points viennent compliquer davantage la situation : (a) l'Écriture est évidemment une chose écrite, en accord avec les règles de la grammaire et dès lors représentative d'une forme mineure de rationalité. Nous devons donc nous demander s'il y a une notion du Verbe divin, de l'Écriture, qui emploierait nécessairement l'instrument de la raison. Et (b), comme nous le verrons, Luther maintient jusqu'à un certain point le principe de l'humaniste qui révèle l'intelligibilité du monde : le monde lui-même est considéré comme un livre à comprendre rationnellement. Les païens, qui n'ont pas d'Écriture, et les Juifs, qui ne reconnaissent pas le ministère de la Parole, se voient accordés une certaine forme - incomplète - de connaissance de la volonté divine. 
Cependant, le moyen par lequel la raison ou la rationalité peuvent être « utilisées » (par opposition à ce dont l'on peut jouir, rappelant la distinction entre uti et frui) n'est pas identifiable à la connaissance incomplète de la volonté divine atteinte par la rationalité naturelle des Grecs. Il s'agit plutôt du produit de l'homme dirigé vers lui-même : par la grâce, l'homme gagne une connaissance de lui-même qui le dirige vers le salut. Cette grâce, obtenue par l'Evangile, manque évidemment chez les Grecs. Si, par la grâce, l'homme accepte sa justification dans une situation de foi, en continuant à savoir (à " posséder le savoir ») qu'il sera toujours un pécheur, alors il peut employer correctement les outils de la rationalité dans un sens herméneutique, c'est-à-dire, sans rien ajouter à son état (à son « texte ») qui ne serait pas

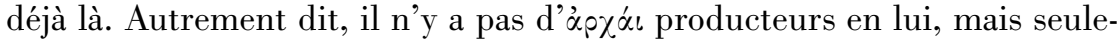
ment des $\dot{\alpha} \rho \chi \alpha \dot{\alpha}$ interprétatifs, intentionnels, qu'il acquiert en propre dans la

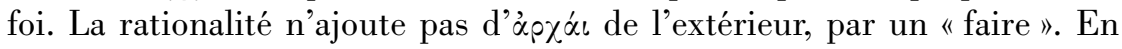
somme, il y a un concept de rationalité chez Luther qui est au-delà de la « raison naturelle "; au-delà du cours ou de la direction naturellement nécessitée par la raison et par le sujet rationnel.

\section{La raison naturelle n'est pas « rationnelle».}

L'extériorité radicale de la raison naturelle à toute véritable connaissance de la doctrine, son manque de "sentiment inscrit dans le cour " (p. 305G [719] ; cf. Romains 2, 15) crée seulement l'illusion d'une compréhension qui se cache à elle-même son impuissance fallacieuse. Certes, la raison naturelle est réfractaire à l'affirmation selon laquelle la volonté divine nécessite toute chose : elle est néanmoins incapable d'affirmer que l'homme nécessite toutes choses. La raison naturelle produit ainsi une position hésitante qui tente de prendre en considération à la fois la volonté infinie de Dieu et la volonté autonome et finie de l'homme. Que ce paradoxe ne puisse être rationnellement maintenu peut, bien entendu, être démontré rationnellement assez aisément. La raison naturelle est incapable d'assimiler rationnellement la doctrine d'un Dieu qui nécessite toutes choses, en affirmant simultanément le libre-arbitre de l’homme : « la raison naturelle est forcée de confesser [...] que le Dieu vivant et vrai soit tel qu'il nous impose la nécessité en vertu de sa liberté » (p. 303G [718]).

Les doctrines sont l'affirmation de la volonté divine prêchée par le ministère de la Parole. La volonté de Dieu en tant qu'elle est prêchée (en tant qu'elle est le contenu des Écritures) est en un sens seulement le "résultat " de la volonté divine : elle n'est pas la volonté divine elle-même. Mais cela est encore une formule inexacte, puisque la parole de Dieu, à la fois en ellemême (la volonté divine) et en tant que message « entendu » (l'Évangile), est toujours identique. Ainsi, elle ne peut pas être rattachée à elle-même dans un 
processus de causalité : elle ne peut pas être un simple « résultat ». En fait, le véritable "résultat» est la foi de l'homme, qui lui permet d'entendre la Parole dans sa clarté : «L'Écriture confesse en toute simplicité la Trinité [et] l'humanité du Christ. [...]. Mais l'Écriture - contrairement à ce que tu imagines - ne dit pas par quelles modalités ces choses sont, et on n'a pas besoin de le savoir » (p. 82G [608]).

La raison crée des objectifs qu'elle satisfait seule, et ensuite affirme qu'elle a su donner une description adéquate de ce qui est contenu dans l'Écriture, mais ces objectifs sont artificiels (ou plutôt "naturels ", c'est-àdire humains) et ne correspondent pas aux limites de la Parole (si on est en droit de parler de limites dans le cas de « das lebendige Wort»). En fait, la raison naturelle obscurcit et confond "naturellement» les délimitations entre la volonté divine et l'objet nécessité par cette volonté - entre la volonté divine prêchée (l'Écriture) et celle qui ne l'est pas. Ainsi, la doctrine (l'affirmation du contenu de l'Écriture, de la volonté divine prêchée par le ministère de la parole, de l'immutabilité et de l'omnipotence de la volonté divine) demeure indifférente aux abus de la raison (la raison « charnelle » d'Érasme lui fait voir des "contradictions" dans l'Écriture, mais son argument se réfute lui-même (p. 333G [733]).

Les tentatives de la part de la raison naturelle en vue de définir l'activité de la volonté divine et simultanément d'affirmer l'existence illusoire du libre-arbitre de l'homme, peuvent ainsi être réduites à la fabrication d'un système rhétorique sans fondement, dont le principal dessein est de se cacher à lui-même ses incohérences rationnelles internes : ainsi que nous l'avons déjà vu, «la raison [...] interprète d'après ses propres déductions et syllogismes les Écritures de Dieu, et les tire dans la direction qu'elle veut » (p. 203G [673]), par exemple en postulant abusivement des figures du discours supposées présentes au sein des Écritures (la diatribe d'Érasme " élude l'argument » par des tropes rhétoriques, p. 293s.G [713s.])

Comme nous l'avons vu, Luther dispose d'un argument contre Érasme afin de prouver que Dieu prévoit, veut, et fait toutes choses selon sa propre volonté immuable, éternelle et infaillible (p. 92G [615]). Cet argument est construit en quatre temps, auxquels nous nous référerons par les lettres (a) à (d).

Le premier argument, (a), est d'ordre rationnel en ce qu'il révèle les auto-limitations de la raison sous un mode performatif. L'argument (b), exposé ici au § II.4, invoque les preuves de l'Ecriture, que Luther souhaite (re-)marquer comme étant claires et transparentes. L'argument (c) est pensé "de l'intérieur », c'est-à-dire que suivant l'exemple des païens, nous avons tous la conscience que Dieu nécessite tout. (Cette conscience peut emprunter au moins deux voies différentes : comme nous le montrons au § II.6, elle peut produire « le mal», autrement dit, une conscience contrefaite ou mal- 
formée du destin ou de la fatalité, et ensuite, comme nous le montrons dans le $\S$ III, elle peut produire une forme plus significative de «conscience » ou d' « éveil » : le désespoir face à la Loi.) L'argument (d) est la plus compliquée des «preuves». Il prend la forme d'un syllogisme pratique : "lorsqu'il promet, il faut que tu sois certain qu'il sait, qu'il peut et qu'il veut réaliser ce qu'il promet. Autrement tu ne l'estimeras pas véridique et fidèle » (p. 96G [619]). Luther ajoute que la conséquence ("autrement ...») équivaut à l'« incrédulité, [l'] impiété suprême et [la] négation du Dieu Très-Haut » (ibid.).

Maintenant, voyons les arguments eux-mêmes.

(a) En premier lieu, Luther démontre que « la raison naturelle elle-même - que [la] nécessité choque et qui s'efforce tant de la supprimer - est forcée de l'admettre, convaincue qu'elle est par son propre jugement, même s'il n'y avait aucune Écriture » (p. 305G [719]). Il construit une sorte de reductio ad absurdum mimétique de la propre diatribe d'Érasme, dans le but (1) de le réfuter en montrant que ses arguments sont identifiables, sinon identiques, à ceux des sophistes, et (2) en radicalisant et exagérant le procédé des sophistes, afin de les «battre à leur propre jeu ». Le jeu rhétorique ici consiste à réfuter un adversaire en s'inscrivant à l'intérieur de son procédé, que l'on parachève ensuite (un des points en commun entre l'argumentaire luthérien et celui d'Hegel ${ }^{4}$ ).

À la question de savoir si toutes les actions sont commandées par Dieu ou non, les sophistes répondent à moitié, en disant que l'agir n'est pas complètement nécessité. Luther traduit cette réponse par la formule qu'il souhaite absurde : «toutes choses arrivent [...] par nécessité de la conséquence, et non par nécessité du conséquent » (p. 93G [616], c'est Luther qui souligne). Une fois cette formule énoncée, Luther démontre que ses adversaires ne sont même pas conscients des fondements de leur propre réponse, à savoir que "toutes choses sont produites nécessairement, mais ces œuvres ainsi " faites" ne sont pas Dieu lui-même !» (p. 94G [617]). En somme, malgré la tentative par la raison de prouver le contraire, l'affirmation originale de Luther, "Dieu nécessite toutes choses », est maintenue. Ici, Luther bloque toute tentative de résoudre le problème en opérant une différenciation entre

4. Le rapprochement entre Luther et Hegel concerne ici la place que chacun accorde à l' "intériorité ». Chez Luther, l'intériorité est produite de manière hétéronome, par la grâce, alors que l'autonomie consiste en sa propre négation : "l'adoration », "l'humilité », etc., que Luther exprime également par des termes tels que "néant» ou "passivité ». Chez Hegel, l'intériorité est produite par le travail de l'Esprit, au moyen de la négativité. Bien sûr, la différence demeure que chez Hegel, ce travail prend fin dans un repli (homecoming) de la conscience qui unit l'hétéronomie (l'Esprit) et l'autonomie (le sujet), alors que chez Luther, un tel processus prend fin par une autre sorte de repli : la connaissance qu'a l'homme de lui-même lui vient de la grâce, et lui prouve alors l'imperfection de sa nature et de la vie temporelle. Il ne peut y avoir d'unité de l'hétéronomie et de l'autonomie. 
pouvoirs divins absolus et décrétés. Exactement comme la proposition sophistique, cette différenciation viendrait se heurter contre un mur : les choses « arrivent [...] par nécessité de la conséquence, et non par nécessité du conséquent »- une pseudo-solution.

Bien que la doctrine soit rationnellement réaffirmée, il devrait être clair qu'elle demeure incompréhensible aux yeux de la raison (naturelle). Ce sont les conditions permettant à la doctrine d'être ce qu'elle est - libre et efficace - qui sont réaffirmées rationnellement, et non pas les croyances fantasmagoriques propres à la raison. Le contenu ou la dynamique de ces conditions (la manière dont la Parole entendue est vraie) ne sont pas réaffirmés. Ce que Luther démontre avec ce premier argument est la reconnaissance rationnelle par la raison naturelle de son propre échec ; cela ne constitue pas en soi une connaissance plus intériorisée de la doctrine.

\section{La clarté de l'Écriture peut être rationnellement saisie.}

Cette démonstration rationnelle est immédiatement suivie par un second argument (b) : Luther apporte les preuves par l'Écriture de son affirmation globale (la volonté divine est immuable, éternelle et infaillible, ainsi Il prévoit, veut, et fait toutes choses selon sa propre volonté, p. 92G [615]) et réitère qu'elle est compréhensible en elle-même et par elle-même, et ne peut être soupçonnée d'obscurité ou d'ambiguïté sur quelque point que ce soit : " "Mon projet se réalisera, ma volonté se fera " (Isä̈e 46:10) - quel écolier ne comprend pas ces mots : "projet", "volonté", " se fera "?" (p. 95G [618], trad. modifiée).

On remarquera une progression nette du premier argument vers le second. Il s'agit d'un mouvement progressif vers une connaissance intériorisée de la volonté divine. Il faut souligner qu'à ce stade l'intériorisation n'est pas encore achevée. Cette progression ne suppose pas non plus des «étapes » d'une séquence qui se déploierait chronologiquement ou logiquement en vue de l'intériorisation à venir. Chaque étape de l'argument représente plutôt une position dont l'hétéronomie absolue ${ }^{5}$ est moindre par rapport à la précédente.

Dans le premier argument, Luther décrit la connaissance de la doctrine (la volonté divine) comme un objet de la raison. Bien que sa radicalisation des arguments rationnels de ses adversaires le conduise à une affirmation « du vrai », elle demeure néanmoins absolument extérieure à son objet. Une reconnaissance rationnelle de l'échec de la raison à réfuter que Dieu néces-

5. "Hétéronomie" et "autonomie" sont employés ici au sens que leur donnait Reiner Schürmann, dans son enseignement. Voir son œuvre posthume Des hégémonies brisées, Mauvezin, T.E.R., 1996, en particulier le chapitre "Kant avec Luther », p. 46ls., et le compterendu par Charles Wolfe, Revue de Métaphysique et de Morale $\mathrm{n}^{\circ} 2$ (avril-juin 1999). 
site toutes choses ne produit, pour la raison, qu'une impasse rationnelle : l'incompréhensibilité. Mais par ailleurs, le caractère compréhensible du type de connaissance présenté dans le second argument signifie que cette connaissance est considérablement moins extérieure, puisqu'elle repose sur un acte ou une potentialité de compréhension chez un sujet de la volonté divine (l'« écolier »).

Cette incompréhensibilité rationnelle absolue et cette capacité de compréhension apparaissent comme deux instances de la rationalité dans le système luthérien, le premier terme produisant la possibilité que le second advienne, et le second étant toutefois essentiellement différent du premier. Ici aussi, nous pouvons parler dans le langage des conditions. Le premier mouvement montre les conditions de la vérité des Évangiles au-delà et contre la vérité de la raison. Le second mouvement a le premier comme condition, et devient lui-même une condition pour le «lieu » dans lequel cette vérité « advient ", à savoir, dans le cœur, intérieurement. Cette seconde condition, comme on le voit avec la figure de l'écolier, est la capacité d'écouter, d'être passif et de laisser la Parole de Dieu être pour elle-même, sine additione.

5. L' incompréhensibilité » de la raison naturelle impose les conditions d'existence de la capacité de compréhension

Comme nous l'avons déjà vu, Luther affirme que vis-à-vis de la volonté divine, on ne pose pas de questions. «Il ne nous appartient pas de chercher à connaître cela ", mais plutôt "d'adorer ces mystères » (p. 292G [712]) ; il suffit de «se taire et de révérer la Majesté de la puissance et de la volonté divines ». Dieu ne nous doit rien, il n'a rien reçu de nous (Romains 11,35), et n'a rien promis sinon ce qu'il a voulu (p. 302G [717]).

«Si je pouvais comprendre par la raison comment un Dieu qui montre tant de colère et d'injustice peut être miséricordieux et juste, je n'aurais pas besoin de la foi. Mais comme cela ne peut être compris, c'est ici que ma foi a l'occasion de s'exercer, lorsque de telles choses sont prêchées et divulguées [...] » (p. 51 / 123G [633], trad. modifiée)

La différenciation qu'opère Luther entre Dieu prêché et non prêché, et entre la connaissance et la compréhension rationnelle, met en place les conditions du passage (qui est en même temps une actualisation, un déploiement) de la connaissance d'une "très claire vérité » (p. 80G [607]), c'est-àdire le contenu de l'Écriture, à sa reconnaissance interne : la foi.

La défaite de la raison naturelle, son échec à comprendre rationnellement la volonté divine laisse une place « ouverte » à l'exercice de la foi, et ce, en vue de l'adoration. Comme nous l'avons vu plus haut, la raison naturelle 
peut rationnellement se démontrer à elle-même son propre échec à répudier la doctrine. Devons-nous conclure que, face au mystère de la volonté divine, la raison provoque sa propre défaite? Ou cette défaite nécessite-t-elle au contraire l'intervention extérieure de Dieu?

La volonté divine impose une limite à notre connaissance rationnelle, qui peut être rationnellement reconnue. Nous l'avons dit, cette situation crée pour la raison une sorte d'impasse rationnelle. L' " adoration » représente un mouvement d'éloignement de la raison. Ce mouvement qui « ouvrait» un espace (pour la foi) est en fait un manque, une privation de la compréhension rationnelle face au mystère de la volonté divine, et non pas un surplus de la raison, une méta-raison ou un argument rationnel qui se prouverait à lui-même qu'il ne peut comprendre une proposition telle que "la volonté divine nécessite toutes choses ». Ce n'est pas une autolimitation de la raison qui définirait ainsi ses propres limites et nierait son propre pouvoir. $L a$ raison ne peut pas se vaincre elle-même. Ou plutôt, la volonté inhérente à la raison naturelle ne peut se vaincre elle-même ${ }^{6}$.

Face à l'incompréhensibilité de la volonté divine, la raison ne peut que continuer de tenter une réinstitution de l'existence illusoire du libre-arbitre de l'homme nécessité par sa propre activité. La raison ne « désespère " pas d'elle-même. Il y a une reconnaissance rationnelle d'un manque de compréhension, mais ce mouvement, qui pose les conditions d'une connaissance intériorisée de la volonté divine, est une « dilution » des pouvoirs de la raison, de sa volonté. Ainsi Luther pourra dire que « rien n'est plus apte à comprendre la Parole que la faiblesse de l'intelligence [sc. la 'compréhension'], car c'est à cause des faibles et vers les faibles que le Christ est venu et qu'il envoie sa Parole » (p. 174G [659]). - Ce n'est pas une fortification, un dernier acte de résistance de la part de la volonté qui la conduit vers la santé ou la reconnaissance, mais un affaiblissement, une atrophie ou, en d'autres termes, une reconnaissance croissante de l'hétéronomie au-delà et contre la volonté initiale d'autonomie, sorte de hubris naturelle.

Plus exactement, ce mouvement représente un changement de direction dans l'inclinaison naturelle de l'activité de la raison, un changement amené, appelé et mû par une force extérieure à lui-même, c'est-à-dire par l'intervention divine. Une force extérieure était requise, mais le « matériel brut » de ce changement de direction, c'est-à-dire la volonté, est présent depuis le début, et une fois que cette "entrée de force » divine a été effectuée, la volonté devient productive (Luther dirait : passive). «[S]i Dieu agit en nous, notre volonté, changée et doucement appellée [...] par l'Esprit de Dieu, veut et agit

6. Ce dernier point est une véritable pierre de touche de la pensée luthérienne : si cette volonté pouvait triompher d'elle-même et ainsi de l'activité de la raison naturelle, il n'y aurait plus de place pour la foi, et plus encore, il n'y aurait pas d'état déchu de l'homme. 
à nouveau avec un pur agrément et une pure inclination, spontanément, sans contrainte» (p. 125G [634]). En d'autres termes, la volonté divine agit en accord avec notre propre nature, nos propres facultés - ici, une faculté rationnelle.

Ce n'est pas par rapport à son objet que la volonté change de direction ; si tel était le cas, nous serions encore dans une sorte de mystique eckhartienne, une pure saisie de soi par soi-même. La volonté change de direction en tant qu'elle ne produit plus son objet ; mais, en un sens plus faible du verbe "produire ", elle produit la condition d'un objet (hétéronome). Toutefois, il faut bien voir que ce n'est pas une autotransformation de la volonté au sens "volontariste»; la transformation est au contraire le fait d'une force extérieure qui l'affaiblit. C'est pourquoi Luther insiste constamment sur le fait que l'Écriture ne pourrait être plus claire. Elle n'a pas besoin d'être produite, ni révélée de l'intérieur, ou rendue compréhensible. Elle demande seulement à être entendue comme elle est, dépouillée de tout élément étranger, de tous les produits de la volonté naturelle et de la raison.

Le changement de direction de la volonté (dans le "cœur» du sujet) procède au moyen de la faculté naturelle, ici, la raison. En d'autres termes, le changement de direction nécessité par Dieu n'est pas une annihilation de la faculté rationnelle - laquelle possède naturellement sa propre direction -, mais un changement qui produit différentes instances de la rationalité, dépourvue de sa volonté jusqu'à ce qu'elle atteigne le niveau de l' "écolier ». C'est un changement qui détourne la raison d'elle-même conçue dans son autonomie et sa puissance "fabricatrice», dans une sorte de conversion au sens littéral, vers cette passivité qui est en même la productivité pure : une capacité de compréhension, d'entendement, d'écoute ${ }^{7}$.

\section{La connaissance de la volonté divine est déjà dans le monde, en l'homme}

Luther en arrive ensuite à son troisième argument (c), qui lui fournit en quelque sorte le matériau pré-chrétien, non-biblique qui vient renforcer sa position : l'insistance de Virgile sur le destin, où nous voyons que dans le peuple, c'est-à-dire le monde, « était restée la connaissance de la prédestination et de la prescience de Dieu, non moins que la connaissance de la divinité » (p. 96G [618]). La Parole est déjà dans le monde. Elle peut être saisie par des voies tortueuses et incomplètes. La Parole est déjà en nous : « les poètes et le peuple, ainsi que leur propre conscience, [la] tiennnent pour tout à fait courante, certaine et vraie " (ibid.).

7. On se souviendra des affinités étymologiques entre acouein et hupacouein, hören et gehorchen, ainsi que entre « entendre » et « entendement». 
Le passage de (b) à (c) suppose que la connaissance de Dieu devient moins absolument extérieure : la Parole est en nous mais son intériorisation n'est pas encore reconnue, donc accomplie.

Il y avait comme une «semence » naturelle de la conscience de Dieu en l'homme, présente dans l'esprit des païens, par exemple. La Parole est déjà dans le monde et peut être saisie par la raison, mais par des voies tortueuses et incomplètes : la connaissance de la prédestination et de la prescience de Dieu était « restée » dans le peuple et chez les poètes. Si cette connaissance ou potentialité de connaissance est dans le monde, cela signifie-t-il la même chose que «en nous»? Nous sommes pleinement dans le monde, mais tout n'est pas présent en nous. Il y a des états naissants, sommeillants, ou encore potentiels. Mais si nous sommes complètement dans le monde, comment peut-il demeurer un "transcendantal » (opposé à la Sachlogik) ? En raison de la seule efficacité de la Parole, répond Luther. Le monde englobe tout, que ce soit la Bible ou nos facultés; cependant, l'activité dépend de la Parole - et nous ne nous donnons pas la Parole à nous-mêmes.

\section{La connaissance comme foi dans la promesse révélée}

La mécanique complexe de l'argument (d) concerne le problème de la condition, mais entendue au sens existentiel : au sens de la possibilité d'existence d'une chose, ainsi que l'expriment des phrases telles que «Je suis la condition pour que cet état de choses advienne », ou encore « La connaissance est une modification de mes états intérieurs ; alors il y a des conditions à cette connaissance ${ }^{8}$.

La connaissance naturelle de l'homme n'est pas l'usage de la raison en tant que "raison guérie » : elle doit toujours demeurer dans l'ombre et en dernier lieu produire l'hubris. Aux yeux de Luther, même Socrate, qui était le meilleur des hommes, ne pouvait que faire de lui-même le principe de sa bonté, et en ce sens oublier qu'il était avant tout dans le monde (ce qui répond partiellement à la question posée plus haut au sujet du rapport entre nous-mêmes et le monde). Le monde, à travers l'expérience que nous avons de lui, et par l'intermédiaire de la théologie naturelle, débouche sur l'hétéronomie, et rien de plus. Ainsi l'argument (c) affirme-t-il aussi que l'homme vit dans un état conditionné au sein du monde, état qu'il doit accepter. La querelle entre Luther et Érasme se résume pour l'essentiel au fait qu'Érasme maintient que nous vivons aussi dans un état conditionnant.

Contre cette affirmation, quelle est la réponse? L'argument (d) en constitue l'étape finale : il s'agit de la foi en tant que croyance intérieure en la

8. Luther avait souligné ce point en référence à Socrate, et à ce que nous pourrions appeler l'établissement d'un humanisme métaphysique, dans son Cours sur l'Épître aux Romains I. 
promesse de Dieu, telle qu'elle est révélée dans les Écritures : «lorsqu'il promet, il faut que tu sois certain qu'il sait, qu'il peut et qu'il veut réaliser ce qu'il promet. Autrement tu ne l'estimeras pas véridique et fidèle, ce qui est incrédulité, impiété suprême et négation du Dieu Très-Haut » (p. 96G [619]). La foi, la connaissance intérieure de la volonté divine, est l'avènement de la reconnaissance intérieure d'une promesse extérieure. Elle apparaît comme étant presque déterminée négativement, mais l'est en fait par une conditionnelle hypothétique, qu'on pourrait présenter ainsi : je devrais croire (faire confiance) en la Parole extérieure, ou je ne serai pas sauvé.

Ce quatrième argument définit la connaissance pour la première fois comme étant absolument corrélative au salut. Ne pas posséder cette connaissance, «ce serait vraiment ignorer Dieu : ignorance par laquelle le salut ne peut tenir, comme c'est bien connu » (ibid.). Le passage de (c) à (d) est un processus d'intériorisation. En fait, il y a ici une redéfinition de la connaissance. Celle-ci ne dénote plus la compréhension correcte d'un objet au sens de l'epistémè, mais plutôt une qualité existentielle produite par les conditions (a) et (c), et par la promesse divine. Elle est dynamique, continue et vivante, plutôt que statique ou "thétique ». La connaissance en tant que compréhension adéquate d'un objet, au contraire, est potentiellement statique. Elle peut être fixée dans les formes intemporelles de la logique. La connaissance du cœur, de la foi, existe en relation avec la temporalité de l'écoute et du temps contenu dans la promesse ("il sait, il peut et il veut réaliser ce qu'il promet »).

La connaissance naturelle de l'homme (à la fois en (b) et en (c)) est une qualité dispositionnelle ou plutôt conditionnelle, une aptitude passive, une capacité à « être saisi par l'Esprit et être rempli par la grâce de Dieu, parce qu'il [sc. l'homme] est créé pour la vie ou la mort éternelles »(p. 128G [636]).

\section{Intériorité et extériorité : la perspective extérieure}

Afin de répondre à la question de savoir « si notre volonté est effectivement active dans les affaires qui relèvent du salut éternel ou subit seulement [sc. de manière passive] l'action de la grâce » (p. 83G [610], trad. modifiée), Luther établit une distinction nette entre la connaissance qu'a l'homme de Dieu et la connaissance qu'a l'homme de lui-même : «il importe donc d'établir une distinction très nette entre la force de Dieu et la nôtre, entre l'œuvre de Dieu et la nôtre, si nous voulons vivre pieusement " (p. 89G [614]).

Comment Luther définit-il notre état naturel ? Il y a une définition négative et une définition positive. Un argument sur ce que nous ne devrions pas être implique son contraire, ce que nous devons être. Quelles facultés possédons-nous ? Sommes-nous passifs jusqu'au moment de notre «éveil »? 
Si tel est le cas, une fois que nous entendons l' "appel », devenons-nous des êtres actifs ? Mais cet état actif ne produit-il pas de nouveaux problèmes, tels que l'hubris, ce cheval sans cavalier?

$\mathrm{Si}$ on essayait de traduire l'argument ontologique pour l'existence de Dieu en termes luthériens, le résultat ressemblerait à ceci. Nous pensons ; nous sommes des êtres de raison; nos facultés rationnelles s'exercent sur quelque chose ; nous possédons la pensée de quelque chose de plus grand que nous, puisque nous avons la notion de grandeur. Dès lors, le « plus grand de tous » doit exister : Dieu. Après Nietzsche, nous pourrions dire : Dieu est garant de la rationalité. En termes plus luthériens, au lieu que la raison s'éloigne de Dieu en montrant la direction opposée, elle s'oriente vers Dieu.

La réaction de Luther à un tel "artefact» concernerait à la fois les Écritures, conçues comme un être hybride entre la raison et l'au-delà de la raison, et la foi. L'entendement ou la raison ne peuvent connaître Dieu, mais la foi peut connaître sa volonté (la volonté divine). On peut alors poser la question en retour : quelle sorte d' « affect » ou de « connaissance » est la foi ? Si elle n'est pas une sorte de connaissance, mais un état de receptivité à la Parole, on est en droit de demander, comme toujours : est-ce un processus temporel ou intemporel ; comment vient-on à la foi, et une fois qu'on y est, peut-elle évoluer? Il semblerait que pour Luther il n'y ait jamais d'arrivée, de terminus, d'accomplissement de la Vérité, de révélation totale. Certes, Luther est chrétien. Il doit donc bien y avoir un telos ou une forme de finalité en vue ${ }^{9}$. À la question concernant la foi, il répondrait alors qu'elle est la conscience de soi-même en tant qu'être qui est sauvé de ses péchés d'une manière extérieure à moi-même. Cette " extériorité ", cette hétéronomie, n'est autre que la volonté divine. Comment connaissons-nous sa volonté ? En un état de passivité : la connaissance de Dieu est proportionnelle à la passivité de notre propre volonté telle qu'elle est reconnue par nous. La volonté, ici, comprend la raison, les œuvres et toutes les autres formes d'activité propres à l'homme.

Nous sommes actifs précisément du point de vue de l'homme charnel, avant que nous ne soyons éveillés et ne devenions passifs. Du point de vue de l'homme spirituel, nous devenons «actifs » dans la foi, c'est-à-dire dans le sens où la connaissance acquise est une connaissance existentielle qui se montre elle-même dans notre vie. Nous savons, dans la fameuse formule de Luther, que dans cet état de connaissance nous continuons d'être pécheurs,

9. Et inversement, la foi est un genre d'intellectus, mais de nature processuelle, dynamique, et sans fin. L'homme dans ce monde n'est pas un être « achevé » pour Luther - c'est le sujet de la Disputatio de homine - car le telos se situe hors de cette vie. Cet inachèvement est d'ailleurs la condition même de la structure simul iustus et peccator, car il permet l'existence d'une temporalité qui n'a pas besoin de se terminer, mais qui peut contenir en une expérience continue des éléments opposés, simultanément : les pécheurs et les justes. 
parce que nous sommes à la fois justifiés (justes) et pécheurs : simul iustus et peccator. Par conséquent, contrairement à un état extérieur à la grâce, il n'y a pas de danger d'hubris, car nous ne nous posons pas comme principe si nous sommes conscients ("prévenus») de notre état continuel de péché. L'activité de la raison naturelle demeure avec nous, et avec elle sa tendance à trouver en elle-même des principes. Mais par la connaissance de soi active propre à l'homme spirituel qui possède la foi, cette tendance est bloquée, essentiellement grâce à la foi en la promesse de Dieu qu'il n'utilisera pas cette même tendance contre nous. C'est pourquoi Luther dit que nous devons clairement différencier entre ce que nous savons de nous-mêmes (la connaissance existentielle) et ce que nous savons de Dieu. Nous ne savons rien de Dieu, sauf la Parole entendue.

Pour Luther, l'argument ontologique serait comme l'exemple par excellence qui témoignerait de notre nature déchue; l'argument refléterait en quelque sorte l'hubris de la raison. Nous savons que Dieu existe en raison du caractère protéiforme et "transformatif » de la foi, et non pas suite d'on ne sait quel syllogisme. Même si nous détenions une telle preuve de l'existence de Dieu, qu'aurions-nous gagné, de son point de vue? Rien, dit Luther, puisqu'un tel savoir n'a rien à voir avec le genre de connaissance qui est propre au salut, le genre décrit sous (d).

Pour résumer, ce que nous avons examiné peut se présenter ainsi :

(i) Le salut est corrélatif à la connaissance, il y est lié.

(ii) La Parole révélée de Dieu est la condition qui met en mouvement tout processus possible de connaissance de Dieu et de toutes les affaires relatives au salut.

(iii) La foi, c'est-à-dire la connaissance intérieure de la volonté divine, est la confiance dans la promesse extérieure. C'est un savoir absolu : «L'Esprit saint n'est pas un sceptique; ce ne sont pas des choses douteuses ou de simples opinions qu'il a écrites dans nos cœurs, mais des assertions plus certaines et plus fermes que la vie même et toute expérience » (p. 77G [605]).

(iv) La connaissance de l'homme a été définie négativement, jusqu'ici, en termes de péchés naturels, comme la direction opposée à l'affirmation naturelle de son libre-arbitre, et comme une capacité passive d'entendre (d' "écoute»). C'est également la "semence» dont nous avons parlé. C'est par cette capacité qu'on descend plus vite la «pente glissante » vers le salut, ou, ce qui revient au même, qu'on permet à Dieu d'entrer en nous plus rapidement. Comme exemples, on peut citer la manière dont « le peuple », ou « les païens », conservent la notion de la prédestination ou la prescience de Dieu comme une idée « tout à fait courante " (p. 96G [618]), mais aussi l' « écolier » qui écoute les Écritures et reconnaît qu'il doit croire afin d'être sauvé.

Que devient la connaissance naturelle de soi dans tout cela ? Que devient la connaissance de soi qui est atteinte par soi-même, sans intervention divine, extérieurement à tout don de Dieu ? À cela Luther répond : quelle 
qu'elle soit, la connaissance de soi naturelle est vouée à l'échec : elle serait une auto-affirmation mégalomane du libre-arbitre de l'homme. L'activité de la raison viendrait obscurcir le fait fondamental que l'homme a été créé par Dieu, et qu'ainsi, l'homme ne peut créer des définitions multiples de ce qu'il est sans tomber dans les erreurs les plus graves.

Nous avons fait un long détour au cours du $\S$ II afin de montrer que quelque chose manquait dans notre délimitation des quatre arguments conduisant au résultat du syllogisme pratique, qui sera présenté au $§$ III. En fait, il manque dans la chaîne d'argumentation de Luther lui-même. Nous entendons parler de connaissance intérieure et extérieure, et de la manière dont elles communiquent, mais tout est défini en termes de connaissance extérieure, c'est-à-dire, en tant que la foi est une réponse à une promesse extérieure. Mais alors, même la foi est définie négativement en termes d'extériorité (la promesse). Dès lors, on est en droit de demander ce qu'est la possession de soi, la pure connaissance de soi qui n'invoquerait aucune extériorité ? La réponse est présentée dans ce qui suit. Dans le texte même, Luther n'offre aucune résolution, car l'hétéronomie absolue, la seule efficacité de Dieu ne peut être contournée ; ainsi il n'y a pas de connaissance de soi en dehors de la grâce. Une telle connaissance ne serait que l'hubris. Luther dira ailleurs que même Aristote connaissait la nature incomplète de l'homme, mais qu'il fut mal interprété par les scolastiques.

III. LA LOI ET LA PROMESSE

\section{Le désespoir face à la Loi}

Vue de l'intérieur, la possession de soi face à la Loi est la connaissance de son propre péché dans le désespoir. De l'intérieur, la possession de soi face à la promesse est la connaissance de soi, en tant que simultanément libérée de la Loi et ainsi sauvée, mais aussi toujours "devant " la Loi, et ainsi pécheresse. Le vieil impératif gnothi seauton, pour Luther, était impossible à satisfaire avant l'arrivée du Nouveau Testament, et cette connaissance de soi survient dans l'espace de la réponse au don de l'Évangile.

Les deux instances de la Parole qui se répondent l'une à l'autre sont la Loi (l'Ancien Testament) et la promesse (l'Évangile). La Loi est donnée pour tenter l'homme, pour lui donner la connaissance du péché, et ainsi lui révéler son incapacité ou son impuissance à engendrer son propre salut. C'est pourquoi on peut dire que la promesse de l'Évangile délivre l'homme de la Loi.

Les étapes que nous avons isolées dans ce « déploiement » se retrouvent sous les formes suivantes dans le texte. 
La Loi de Moïse ne dit pas : «Tu as la force ou la puissance de choisir », mais "Choisis, garde, fais " (p. 215G [678]). La diatribe d'Érasme, toute pleine qu'elle est de distinctions métaphysiques entre acte et puissance, interprète mal le caractère impératif de l'Ecclésiastique, rendu plus opaque aussi par le fait que les Hébreux utilisent l'indicatif futur ("tu ne tueras pas ...») à la place de l'impératif. Il ne s'agit pas de ce que peuvent les hommes, mais bien de ce qu'ils doivent (p. 210G [677]), et quant aux distinctions métaphysiques sus-mentionnées, «les paroles du prophète incluent aussi bien l'acte que la puissance » (p. 399G [762]). L'essentiel est que c'est "par la Loi » que l'on prend « connaissance du péché» (Romains 3,20, cité p. 213-214G [677], c'est nous qui soulignons). Comment la Loi nous mène-t-elle à la connaissance du péché, autrement dit, de notre finitude?

«Dieu nous tente, afin de nous conduire par la loi à la connaissance de notre impuissance [...] L'Écriture arrête que l'homme est corrompu et captif, qu'en outre il méprise et ignore orgueilleusement sa corruption et sa captivité ; c'est pourquoi elle le tire et le réveille par ces paroles-là, afin qu'il reconnaisse, même par expérience certaine, qu'il ne peut rien de ces choses " (p. 205-206G [673674]).

Si l'homme n'est pas ainsi «animé » ou "réveillé », son «libre-arbitre, par ses propres forces, ne peut rien exercer sinon le "service armé" du péché ; au point où la foi elle-même, que l'on pense lui être donnée en aide, lui a été insupportable et l'a rendu encore plus pécheur, du moment qu'il faisait le service armé sous ses ordres » (p. 345G [738]). On commence à voir pourquoi l'homme aurait besoin d'être délivré de la Loi, par l'Évangile (par exemple, p. 112G [628]) : "ceux qui s'emploient avec le plus de zèle aux œuvres de la Loi sont ceux qui l'accomplissent le moins, d'autant qu'il leur manque l'Esprit, qui seul peut l'accomplir » (p. 406G [766], trad. modifiée).

C'est ainsi, par exemple, que les Juifs ne peuvent que rester pécheurs : " ils sont transgresseurs de la Loi, parce qu'ils ne sont pas Juifs " en Esprit", bien mieux : parce qu'ils résistent obstinément à la justice de la foi » (p. 394G [760]). On a vu plus haut que c'est par la Loi que l'homme prend connaissance du péché, mais pis encore, "la Loi est la puissance du péché » (1 Corinthiens 15,56), « le montrant mais ne l'enlevant pas »; ainsi la Loi « rend la conscience coupable devant Dieu »(p. 424G [773], c'est nous qui soulignons).

Dès lors, la notion à laquelle nous avons parfois fait allusion, de « désespoir devant la Loi », commence à se préciser : "la parole de la Loi n'est prononcée que sur ceux qui ne sentent pas le péché et ne le reconnaissent pas [...], la parole de la grâce ne vient que vers ceux qui, ressentant le péché, sont affligés et tentés par le désespoir " (p. 228G [685]). Sans la promesse divine de la grâce, autrement dit, de la "miséricorde, la vie, la paix et le salut " même, sans cette promesse qui permet de "redresser " les consciences 
« affligées par le sens du péché et terrifiées par la crainte de la mort et du jugement» (p. 227G [683]), comment pourrions-nous concevoir le pardon et l'espérance ? C'est ainsi que le désespoir face à la Loi, qui est la "puissance » même du péché, mène au tournant vers l'intériorisation, qui sera décrit au $\S 2$.

\section{La connaissance de la Loi}

Dans l'Ancien Testament, la Loi est donnée. La connaissance de la Loi est un objet radicalement extérieur, qui suppose que le salut requiert les œuvres, c'est-à-dire l'activité. Par les œuvres, l'homme prend connaissance de son état de pécheur ; il comprend ainsi l'impossibilité ou l'incapacité de satisfaire aux impératifs de la Loi par les seules œuvres, puisque l'activité des œuvres éloigne l'homme de la foi, de la volonté divine et du Saint-Esprit. La culpabilité et le désespoir surviennent avec la reconnaissance intérieure de l'impuissance à satisfaire les exigences de la Loi. Ce désespoir et cette reconnaissance préparent les conditions nécessaires à la réception du don de l'Évangile.

Il peut être utile de remarquer la similarité démonstrative entre l'argument luthérien exposé au $§$ II, concernant le balancement entre la connaissance extérieure et la reconnaissance intérieure de l'échec à connaître par la raison, et l'argument présenté ici, concernant la connaissance extérieure de la Loi et la reconnaissance intérieure de l'inaptitude à satisfaire la Loi par les œuvres. Le second mouvement de ce balancement est plus précis que le premier, et en fait il y a plus qu'une simple ressemblance. Le balancement dans l'argumentation est repris et approfondi, car l'argument a été déplacé du concept général du contenu de l'Ecriture vers une définition plus précise de ce contenu. L'Écriture est en effet divisée en deux parties : la Loi (l'Ancien Testament) et la Promesse (le Nouveau Testament). «L'œuvre de Moïse et du législateur [...] consiste à montrer à l'homme, par la Loi, sa misère, afin que, l'ayant de la sorte brisé et confondu dans la connaissance qu'il a de lui-même, elle le prépare à la grâce, l'envoie au Christ, et qu'il soit ainsi sauvé » (p. 218G [679]).

Ici, la connaissance de soi est extérieure dans l'état de péché ; elle est moins radicalement extérieure dans l'état de désespoir, ce qui constitue la première ouverture, si petite soit-elle, d'un espace pour la conscience de soi.

Le désespoir n'est plus simplement une forme de connaissance, mais déjà un certain genre de conscience de soi, de comportement dirigé par une finalité. On peut maintenant relire d'une nouvelle manière le passage cité plus haut (§ II.2), sur la volonté qui est comme une monture placée entre deux cavaliers : «Si c'est Dieu qui la monte, elle veut aller et elle va là où Dieu veut [...]. Si Satan la monte, elle veut aller et elle va là où Satan veut » (p. 127G [635]). Il y a une force dans l'homme qui l'oblige à vouloir quelque chose, que ce soit le bien ou le mal. C'est cette force qui peut être « sellée » ou « montée » par un cavalier, qui n'est précisément pas l'homme lui-même. C'est seu- 
lement dans l'état de désespoir que l'homme peut abandonner complètement cette force innée ; alors le salut et la connaissance peuvent avoir lieu.

\section{Remarque}

On peut voir ce mouvement comme étant très «nietzschéen 》: (a) l'homme préférerait ne rien vouloir plutôt que de ne pas vouloir, et (b) l'idée d'une volonté qui se placerait contre la volonté, et produirait ainsi une positivité plus élevée. Cependant, chez Luther, les deux moments (a) et (b) prennent des allures fort différentes. D'abord, (a) ce n'est pas l'homme qui «veut», mais plutôt Dieu ou Satan; l'homme n'a pas le choix en cette matière. Ensuite, (b) la négation de la négation ne produit pas une positivité supérieure ; la volonté humaine s'efface, un point c'est tout. Cet effacement produit une rationalité dénuée de volonté (et quant à la volonté divine, nous ne pouvons pas la connaître rationnellement). Néanmoins, et sans entrer dans des querelles très éloignées de l'objet qui nous occupe ici, à moins de donner une interprétation très « dialectique » du point (b), on peut considérer cet « effacement » comme signifiant l'absence même de toute dialectique. En effet, il est difficile de parler d'une réconciliation interne des contraires, motivée par une fin sous-jacente ou totalisante, un "grand projet ». Si un affect « finalisé » (la volonté humaine dirigée par l'hubris) est annulé par un autre affect « anti-finaliste » (le désespoir, l'éminence d'un manque de déterminations, etc.), on voit alors qu'une pluralité de forces sont en jeu.

C'est pourquoi on peut considérer Luther comme l'initiateur ou le découvreur d'une « zone » nommée « conscience de soi », une zone que nous percevons comme étant active, alors que chez Luther elle est pensée comme étant passive. La foi est une sorte de connaissance, processuelle et cependant certaine. Elle n'est pas évidente comme le serait l'epistémè mais certaine au sens de la "certitude de soi », à laquelle appartient d'ailleurs la conscience que notre être dans cette vie est incomplet. On remarquera en passant que c'est pour cette raison que Luther peut présider une religion si «mondaine " : une fois que la connaissance est adéquate (pas à son objet mais au sujet, à son "état " dans le monde ; ainsi nous avons pu parler de "connaissance existentielle»), rien de plus ne doit changer. Nous devons continuer nos œuvres, du moment que nous savons qu'elles n’ont rien à voir avec le salut. Une fois que nous sommes dotés d'une connaissance de soi juste, la volonté peut à nouveau vouloir, elle a été « mise à sa place ».

3. La connaissance de soi (intériorité et extériorité : la perspective intérieure)

Le désespoir extrême face à la Loi met en place les conditions de la grâce. L'Évangile est la délivrance de la Loi de Moïse. La promesse impose que la 
proposition de l'homme devrait être «Je devrais croire» et non pas «Je devrais œuvrer ». La foi est la confiance en cette promesse, et ainsi, elle est le tournant vers l'intérieur (l'intériorisation), c'est-à-dire la production de la conscience de soi, qui a lieu dans la Loi. Le mouvement d'intériorisation est par là même le tournant vers la Loi, la loi divine achevée par la grâce. L'homme ne peut pas satisfaire la Loi ; du point de la vue de la raison « charnelle », c'est-à-dire humaine, le libre-arbitre est bafoué, on « se moque de l'homme par un commandement impossible [à observer] ", alors que du point de vue luthérien, "cet homme-là précisément a été averti et stimulé pour qu'il voie son impuissance» (p. 212G [676]). Par sa passivité, «cet homme » reçoit la volonté divine, et entend la promesse faite par Dieu du salut face à la Loi. La foi est bien la confiance en la promesse, au sens où réaliser que "Je ne peux pas satisfaire la loi " (affirmé dans un état de désespoir) conduit à : «mais je peux reconnaître que j’ai été sauvé » (je peux reconnaître que j'ai totalement soumis ma volonté a celle de Dieu).

Luther avait affirmé que « si Dieu agit en nous, notre volonté, changée et doucement appellée [...] par l'Esprit de Dieu, veut et agit à nouveau [...] spontanément, sans contrainte » (p. 125G [634]). Autrement dit, Dieu produit en nous une nouvelle spontanéité « douce », et il y a alors un véritable renouveau de la Loi, à la fois dans la loi divine telle qu'elle est accomplie par la grâce, et "face à la Loi ». Ces deux conditions réunies produisent la formule simul iustus et peccator - dans notre vocabulaire " gnoséologique ", la connaissance simultanée de soi-même en tant que pécheur et que sauvé.

Ainsi, l'objet de la connaissance de soi est la foi, c'est-à-dire la foi telle qu'elle s'exprime dans l'affirmation « Je veux la connaissance de mon salut ». La volonté ici est le siège d'une capacité passive, une faculté entièrement vouée à Dieu, ou mieux, elle est la volonté de devenir réceptif à la grâce, par le désespoir ou la souffrance ${ }^{10}$. Enfin, il s'agit de la connaissance que nous sommes à la fois pécheurs et sauvés. Mais de quelle nature est cette connaissance? Elle est la foi et la confiance en la promesse que la volonté divine nécessite toutes choses, promesse d'un monde à venir, du moment d'une reconnaissance de soi absolue.

$$
* *
$$

«Mais l'homme ne peut s'humilier à fond, jusqu'à ce qu'il sache que son salut ne dépend pas du tout de ses forces, de ses desseins, de ses attachements, de sa volonté et de ses œuvres, mais bien plutôt de l'arbitrage, du dessein, de la volonté

10. Luther emploie les deux expressions indifféremment, mais si nous le relisons à l'horizon de l'idéalisme allemand, nous pouvons projeter sur son œuvre une distinction entre le désespoir qui serait d'ordre transcendantal et la souffrance (Leiden) qui marquerait la prise de conscience de soi et du monde. 
et de l'œuvre d'un autre : de Dieu seul [...]. En effet, aussi longtemps qu'un homme est persuadé qu'il peut ne serait-ce qu'une toute petite chose pour son salut, il reste dans la confiance en lui-même et ne désespère pas tout à fait de lui : il ne s'humilie donc pas devant Dieu, mais présume ou espère [...] un lieu, un temps ou une œuvre par quoi il parviendra finalement au salut. Mais celui qui n'a aucun doute sur le fait que tout dépend de la volonté de Dieu, celui-là désespère tout à fait de lui-même, ne choisit rien, mais attend Dieu qui agit ; celui-là est très proche de la grâce, " pour être sauvé " (1 Tim. 1:15) " (p. 122G [632-633]).

Reprenons les deux questions posées au départ (§ I.2) : «Que savonsnous ? ", et «Comment le savons-nous ? ». La réponse à la première question est : les faits et le contenu des Écritures, qui nous conduisent vers la Loi et la Promesse en nous affirmant que nous sommes des pécheurs et que nous serons sauvés. La réponse à la deuxième question est : par le Ministère de la Parole, qui nous conduit aussi vers la Loi et la Promesse, mais par le désespoir et la foi. Ainsi que le montre le passage ci-dessus, l'état de désespoir produit une suspension de la volonté qui mène à la passivité et à la réceptivité ; c'est alors que, la raison orgueilleuse étant dissipée, la grâce devient possible. Le problème de l'existence simultanée de la connaissance d'un objet extérieur (la clarté des Écritures) et de la connaissance intérieure dans la foi, ainsi que toutes les tentatives de décrire les étapes de cette existence par des formules tant logiques ("posées») que temporelles ( engendrées »), apparaissent alors comme autant d'apories. En réalité, tout se joue dans le passage de la clarté extérieure des Écritures à la clarté intérieure de la foi. C'est précisément en «niant » l'autonomie du sujet (la raison naturelle), en affirmant son état d'hétéronomie et sa passivité (la raison redirigée et affaiblie dans la foi), que Luther constitue l'espace de la conscience de soi.

\section{(traduit par Christian NAdEAU)}

Résumé : En étudiant le rapport entre connaissance, foi et volonté dans le Traité du serf-arbitre de Luther, il s'agit de montrer comment il délimite un espace correspondant à la «conscience de soi " moderne, mais qu'il définit comme un état de passivité pure, d'hétéronomie par apport à la Loi divine et non d'autonomie de la raison ou de la volonté. Cette passivité pourtant source de spontanéité correspond à l'état que Luther appelle "simultanément justifié et pécheur ».

Mots-clés : Volonté. Foi. Raison. Hétéronomie.

Abstract : By examining the relation between knowledge, faith and will in Luther's Bondage of the Will, our aim is to show how he delimits a space corresponding to modern "self-consciousness", which he however defines as a space of pure passivity, of heteronomy in relation to the divine Law rather than autonomy of reason or the will. This passivity which is nevertheless a source of spontaneity corresponds to the condition Luther describes as " simultaneously justified and a sinner 》.

Key words : Will. Faith. Reason. Heteronomy. 